\title{
Using Fluorescence Recovery After Photobleaching to Study Gap Junctional Communication In Vitro
}

\author{
Maria Kuzma-Kuzniarska $1, *$ \\ Email maria.kuzmakuzniarska@gmail.com \\ Clarence Yapp 2 \\ Philippa A. Hulley 1 \\ 1 Nuffield Department of Orthopaedics, Rheumatology and Musculoskeletal Sciences, University \\ of Oxford, Windmill Road, Oxford, OX3 7HE UK \\ 2 Structural Genomics Consortium, University of Oxford, Oxford, UK
}

\begin{abstract}
Fluorescence recovery after photobleaching (FRAP) is a microscopy-based technique to study the movement of fluorescent molecules inside a cell. Although initially developed to investigate intracellular mobility, FRAP can be also used to measure intercellular dynamics. This chapter describes how to perform FRAP experiment to study gap junctional communication in living cells. The procedures described here can be carried out with a laser-scanning confocal microscope and any in vitro cultured cells known to communicate via gap junctions. In addition, the method can be easily adjusted to measure gap junction function in 3D cell cultures as well as ex vivo tissue.
\end{abstract}

\section{Keywords}

Fluorescence recovery after photobleaching

Confocal microscopy

Gap junctions

Intercellular communication

Calcein

In vitro

Monolayer

Tenocytes

Fluorescence recovery curve

Mobile fraction percentage

AQ1

\section{Introduction}

Fluorescence recovery after photobleaching (FRAP) permits quantitative assessment of the mobility of fluorescent molecules. FRAP is used to address questions related to protein localisation and interactions, mobility of molecules within cell compartments as well as organelle dynamics. The principle behind FRAP is simple, fluorescently labeled molecules are irreversibly bleached in a 
well-defined area, called the region of interest (ROI). Following the bleaching, unbleached molecules from outside the ROI, diffuse into the bleached area. This movement is observed as an increase in fluorescence intensity (fluorescence recovery) in the ROI. The rate of fluorescence recovery reflects the mobility of molecules and the analysis of the recovery curve yields quantitative information about the process $[1,2]$.

Although FRAP was initially developed to study intracellular dynamics, Wade et al. adjusted the technique to measure the transfer of fluorescent molecules between cells. They extended the analysis from a small region to the whole cell and showed that it is possible to perform multiple fluorescence measurements of the same living cell. By choosing a fluorescent dye that can easily be transferred through gap junctions, they were able to assess the movement of fluorescent molecules between cells and as a result created a new technique to study gap junctional communication [3]. One of the main advantages of FRAP over other methods used to study gap junction function, such as scrape loading and microinjection, is that FRAP experiments are noninvasive, thus all procedures are performed without disrupting cell integrity. FRAP is also regarded as less time consuming when compared to microinjection, and less technically demanding when compared to dual whole-cell patch clamp [4].

A FRAP experiment to study gap junctional communication can be divided into three stages (Fig. 1 ). First, prebleach imaging is performed in order to gain information about the initial fluorescence level. Next the cell of interest is selected and bleached with a high-powered laser. Finally, post-bleach fluorescence recovery is monitored using the same laser but at low power. It is important to note that for the recovery to occur, the cells not only need to display functional gap junctions but enough cell partners must be present to exchange the fluorescent molecules (see Note 1 ) [5]. The fluorescent labeling can be performed using any fluorescent dye that can be transferred via gap junctions. Among different tracers calcein acetoxymethyl (AM) ester can be recommended. Calcein-AM is converted to calcein after acetoxymethyl ester hydrolysis by intracellular esterases. Upon the conversion to membrane impermeable calcein emits green fluorescence. Calcein is well retained by viable cells, yields high fluorescence, and can be effectively transferred between cells through gap junctions [6].

\section{Fig. 1}

A schematic representation of the principle of FRAP experiments. (a) In a typical FRAP experiment a population of intracellular molecules is fluorescently labeled. Next the fluorescent molecules in a small area, called the region of interest (ROI), are permanently bleached using a high power laser beam. Finally fluorescence recovery to the ROI is measured over time. The recovery in the ROI is based on the diffusion of the unbleached molecules into the bleached region of interest and provides information about the mobility of fluorescence molecules. In experiments employing the FRAP method to study gap junctions all fluorescently labeled molecules within a cell are permanently bleached, thus the region of interest is extended to the whole cell and the exchange of molecules via gap junctions between a bleached cell and the unbleached neighboring cells is responsible for the fluorescence recovery. (b) Two parameters can be used to describe fluorescence recovery in a bleached cell: recovery time and mobile fraction. $F$ initial -fluorescence intensity before bleaching, $F_{0}$-fluorescence intensity immediately after bleaching, $F_{\mathrm{m}}-$ mobile fraction (fraction that contributes to the recovery), $F_{\mathrm{i}}$-immobile fraction (fraction that does not contribute to the recovery) $t_{1 / 2}-$ half time recovery 


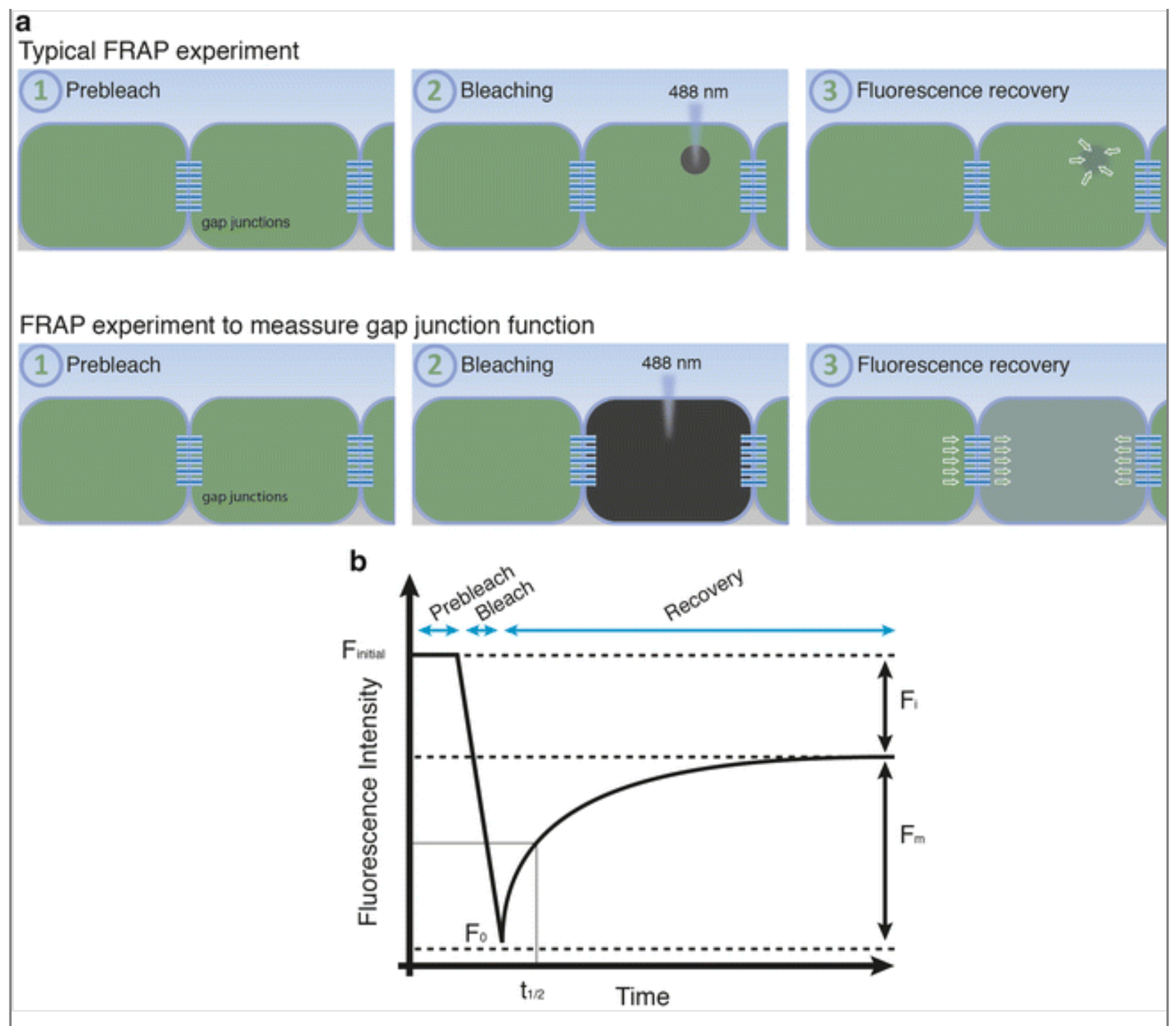

This chapter outlines how to set up, perform, and analyze FRAP experiments in cultured cells to assess gap junction function.

\section{Materials}

\subsection{Cell Culture}

1. Human hamstring tendon cells (tenocytes) isolated by standard explant culture method (see Note 2 ).

2. Cell culture media: Dulbecco's Modified Eagle Medium supplemented with $10 \%$ fetal bovine serum (FBS). Store at $2-8{ }^{\circ} \mathrm{C}$.

3. Glass bottom dishes 35-mm with \#1.5H $(170 \mu \mathrm{m} \pm 5 \mu \mathrm{m}) \mathrm{D} 263 \mathrm{M}$ Schott glass.

4. Incubator at $37^{\circ} \mathrm{C}, 5 \% \mathrm{CO}_{2}, 90 \%$ humidity.

5. Water bath at $37^{\circ} \mathrm{C}$. 


\subsection{Labeling with Calcein-AM}

1. $1 \mathrm{mM}$ calcein acetoxy-methylester (AM) stock solution dissolved in DMSO.

2. Serum-free culture medium: Dulbecco's Modified Eagle Medium with no supplements.

\section{Methods}

\subsection{Cell Culture}

1. Grow tenocytes in cell culture medium.

2. Passage cells by scraping at $70 \%$ confluency.

3. Seed tenocyte cells at a concentration of approximately $2 \times 10^{5}$ cells per well in $2 \mathrm{~mL}$ of medium onto $35 \mathrm{~mm}$ glass bottom dishes and culture to provide confluent cultures within 2-3 days (see Note 3 ).

\subsection{Labeling with Calcein-AM}

1. Thaw calcein-AM stock solution and prepare a $4 \mu \mathrm{M}$ working solution by diluting 1:250, e.g., $4 \mu \mathrm{L}$ of calcein-AM stock solution per $1 \mathrm{~mL}$ of warmed serum-free culture medium (see Note 4 ).

2. Remove media from tenocytes and wash cells once with warmed serum-free culture medium.

3. Remove the serum-free culture medium and replace with $1 \mathrm{~mL}$ of calcein-AM working solution.

4. Incubate the cells with calcein-AM for $15 \mathrm{~min}$ at room temperature or alternatively place the dishes in the incubator at $37^{\circ} \mathrm{C}$.

5. Cover to protect from light.

6. Wash the cells three times with pre-warmed serum-free medium to ensure that no calcein-AM is present in the medium.

7. Fill the dish with $1 \mathrm{~mL}$ of pre-warmed serum-free medium and proceed immediately with FRAP (see Note 5 ).

\subsection{Setup for Fluorescence Recovery After Photobleaching}

1. Perform procedure on a confocal laser scanning microscope (e.g., Zeiss LSM7 with Zen 2009 software) equipped with a $20 \times 0.5$ numerical aperture objective, a $488 \mathrm{~nm}$ argon-ion laser and an incubator chamber capable of maintaining temperature at $37^{\circ} \mathrm{C}$. 
2. Mount the glass bottom dish with labeled cells on a motorized scan stage with an incubator chamber maintaining $37^{\circ} \mathrm{C}$.

3. Focus the microscope on the sample using epi-fluorescence mode with a GFP or similar filter cube.

4. Acquire one pre-bleach scan at low power to record the initial fluorescence intensity of the cell.

5. In order to accomplish complete photobleaching of calcein, adjust settings to irradiate the ROI four times using a pixel dwell time of $50 \mu$ s with argon-ion laser power set to the maximum (see Note 6 ).

6. Immediately after photobleaching the ROI, acquire a rapid time series (e.g., $5 \mathrm{~s}$ intervals for $4 \mathrm{~min}$ ) to record the recovery of calcein. Ensure that the duration and laser power is set so that complete recovery is observed without excess photobleaching (see Notes $7-9$ ).

7. Once appropriate conditions for photobleaching and imaging have been established, use the saved settings to perform FRAP experiments.

\subsection{Performing the FRAP Measurement}

1. Focus the microscope on the sample using epi-fluorescence mode with a GFP or similar filter cube.

2. Choose an appropriate region for photobleaching by manually drawing a ROI around selected cells.

3. Draw additional reference regions of interest around a cell not selected for bleaching and a region outside the cells to provide measures for non-bleached and background signal.

4. Photobleach the ROI using the previously established parameters.

5. Following photobleaching, record a rapid time series (e.g., $5 \mathrm{~s}$ intervals for $4 \mathrm{~min}$ ) to record the recovery of calcein of fluorescence recovery using $1 \%$ of the laser power (see Note 10 ). An e of a FRAP experiment and a representative fluorescence recovery curve can be found in Fig. 2

\section{Fig. 2}

An example of a FRAP experiment using human tendon cells (tenocytes). (a) Calcein tenocytes were subjected to photobleaching using a $488 \mathrm{~nm}$ laser at $100 \%$ power. Subseq time-lapse series was recorded using $1 \%$ power. The recovery is shown in images at $<1 \mathrm{mir}$ min post-bleaching. As illustrated above, recovery depends on the number of adjacent cells the number of connections formed between cells. Cells grown at low density will fc cell-to-cell contacts and therefore will be less likely to communicate via gap junction subjected to photobleaching the fluorescence recovery will be impaired when compared to $c$ confluent monolayer. (b) An example of a FRAP curve obtained for a cell (confluent mo culture) undergoing photobleaching and subsequent fluorescence recovery. Changes in fluo: 
intensity over time are shown. After a sharp decline in fluorescence the intensity increase gap junctional communication
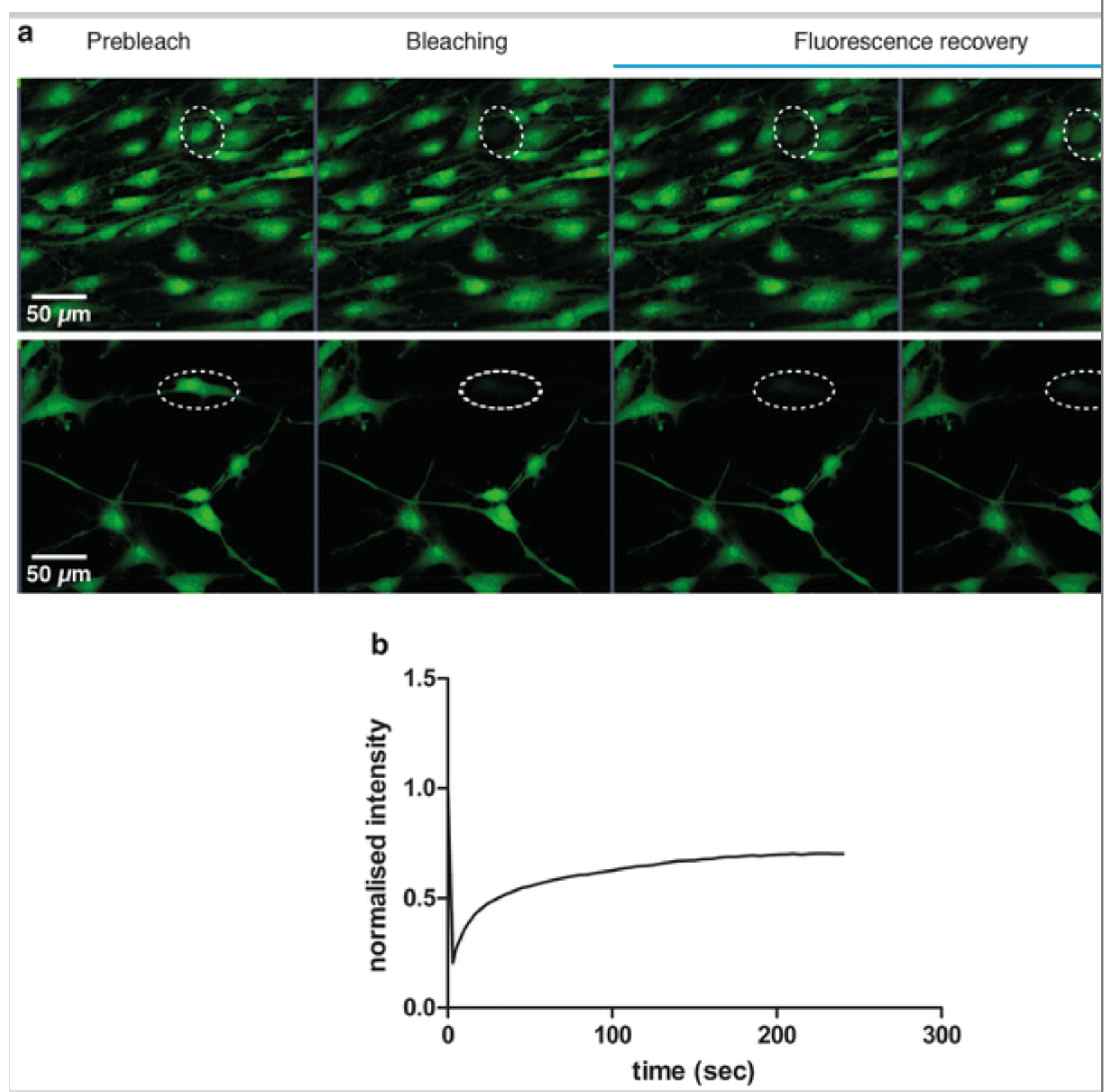

6. Using software settings, the detector gain can be adjusted to image cells with varying labeling intensities.

7. It is possible to inhibit the recovery by using gap junction inhibitors (see Note 11 ) [5, 7].

8. Common problems associated with FRAP experiments, based on the type of recovery curve obtained in the experiment, are shown in Fig. 3 .

Fig. 3

Types of curves encountered when measuring gap junctional communication using the FRAP method and common problems associated with them. (a) Typical recovery curve with a sharp increase in fluorescence intensity after bleaching followed by a plateau. Common issues include (b) Recovery without a plateau which indicates that dye is still 
present in the medium and taken up by the cell during the course of the experiment. (c) A decrease in fluorescence intensity over time in the recovery stage that indicates bleaching of the sample during the recovery imaging or the sample going out of focus. (d) Initial recovery followed by a sharp decline in fluorescence indicates that the sample has dried out or cell death
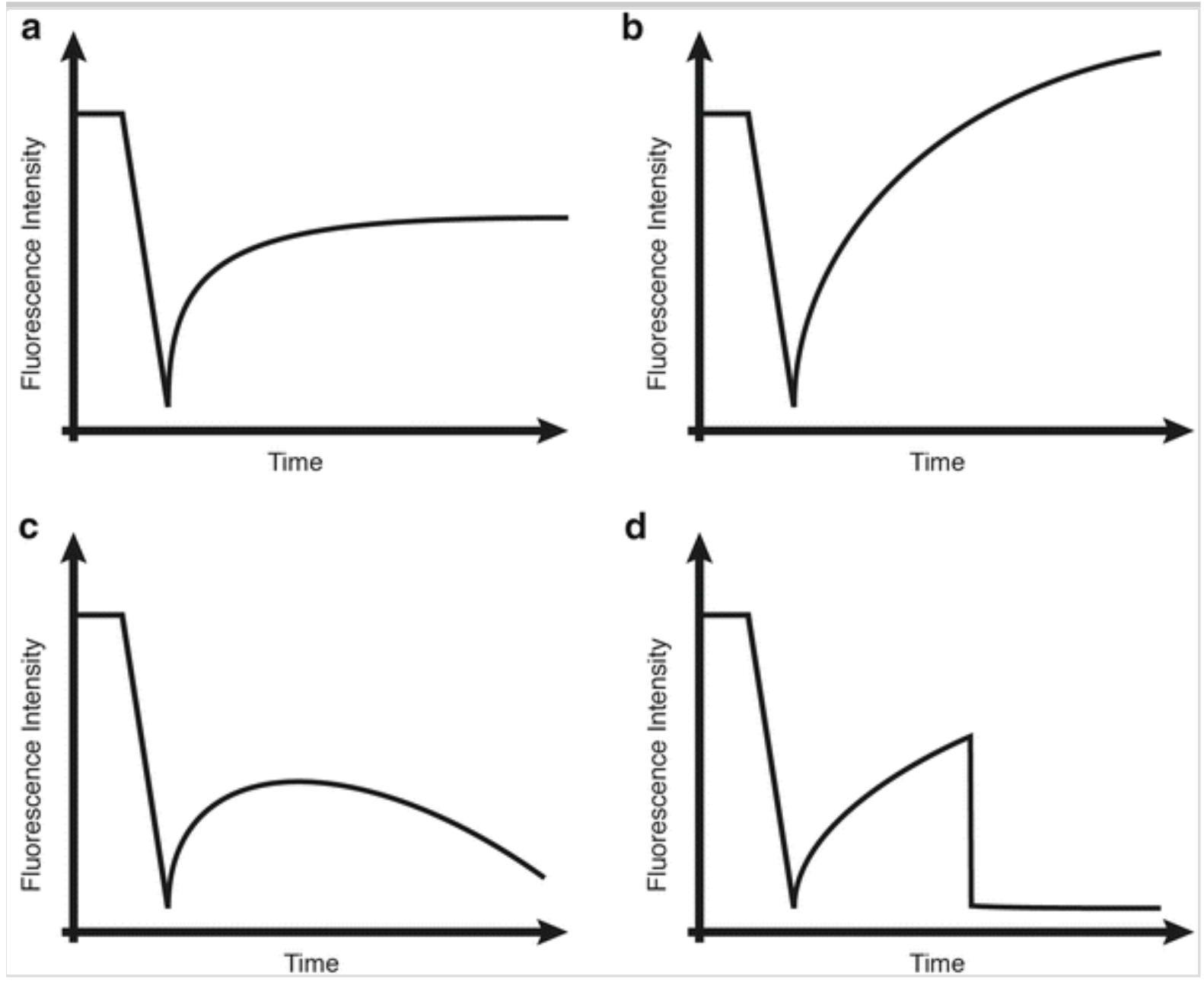

9. Collect data for at least ten cells per treatment for each experiment.

10. In order to obtain reproducible data it is important to keep the basic imaging settings the same in all subsequent experiments and to not adjust bleaching and recovery conditions between experiments.

\subsection{FRAP Analysis}

1. Analysis of fluorescence recovery in bleached cells can be assessed by calculating the mobile fraction $\left(F_{\mathrm{m}}\right)$ percentage.

2. Software analysis, e.g., (Zeiss, ZEN 2009) can be used to identify the fluorescence intensities of the cell at full recovery $\left(F_{\mathrm{m}}\right)$, immediately after photobleaching $\left(F_{0}\right)$, and before photobleaching $\left(F_{\text {initial }}\right)$. 
3. The mobile fraction percentage is then calculated using the equation:

$$
\text { mobile fraction percentage }=\left[\frac{\left(F_{\mathrm{m}}-F_{0}\right)}{\left(F_{\text {initial }}-F_{0}\right)}\right] \times 100
$$

\section{Notes}

1. Cells in low-density cultures form fewer cell-to-cell contacts and therefore are likely to show impaired gap junctional communication when compared to high-density cultures. The low-density cultures can be used as a simple control, i.e., fluorescence recovery should not be seen in single cells not connected to other cells. Other methods include the use of a cell type that does not communicate via gap junctions, such as the HeLa cell line, or a gap junctional inhibitor, e.g., glycyrrhetinic acid.

2. Any adherent cells that communicate via gap junctions can be used for this protocol. Both primary cells and cell lines derived from human and other species are suitable.

3. When comparing different experimental conditions it is important to use cultures with comparable levels of cell confluency.

4. Calcein is a membrane-impermeable hydrolysis product of calcein-AM that is also commonly utilized as a viability indicator. Therefore calcein labeling can also be used to control for the viability of cells subjected to FRAP.

5. It is possible to stain monolayer cultures, 3D cell cultures and small pieces of tissue with calcein-AM. The protocol for staining is the same although it might be necessary to increase the time of incubation with the dye. In addition the samples might require an immobilization step to prevent problems with going out of focus during the experiment. It is also crucial to prevent the samples from drying out during the labeling step as well as at any other step in the protocol.

6. Both photobleaching and fluorescence imaging of calcein make use of an argon-ion laser $(488 \mathrm{~nm})$ and a piezomultiplier tube for detection of fluorescence between 500 and $550 \mathrm{~nm}$.

7. To obtain a suitable laser power setting for minimal photobleaching, the average intensity of the bleach region can be monitored over time until a plateau is reached (Fig. 3a). If this does not occur (e.g., Fig. 3c), the laser power during image acquisition may be too high. If lowering the laser power results in a dark or noisy image, the pinhole should be opened until sufficient photons can be detected.

8. In tenocytes, the initial recovery of calcein is rapid. In order to model the recovery as accurately as possible, the time-lapse acquisition must record as much of the initial stages of recovery as possible.

9. A significant portion of the recovery may be missed when the microscope is changing modes between bleaching and time-lapse acquisition modes. Therefore, in order to reduce this changeover time, the zoom can be increased to a value so that the cell is still within the field of view. 
10. Scanning is performed unidirectionally with fast scan speed (e.g., 9 on the Zen 2009 platform) and low averaging (e.g., setting of 1 on the Zen 2009 platform) to enable fast recording of the fluorescent recovery. Post-bleaching acquisition should be performed every $5 \mathrm{~s}$ for up to $4 \mathrm{~min}$.

11. Gap junction communication can be blocked by incubating the cells with $100 \mu \mathrm{M} 18$ $\beta$-glycyrrhetinic acid (GA) in DMEM-F12 supplemented with $10 \%$ FBS. The inhibitor should be present during the whole course of the FRAP experiment. GA stock solution can be prepared in dimethyl sulfoxide (DMSO) and stored at $-20{ }^{\circ} \mathrm{C}$.

\section{Acknowledgements}

Our studies to develop the published methods were supported by Orthopaedic Research UK, Arthritis Research UK, the Oxford NIHR Musculoskeletal Biomedical Research Unit and the Botnar Research Centre Bioimaging Small Research Facility, University of Oxford.

\section{References}

1. Reits EA, Neefjes JJ (2001) From fixed to FRAP: measuring protein mobility and activity in living cells. Nat Cell Biol 3:E145-E147

2. Staras K, Mikulincer D, Gitler D (2013) Monitoring and quantifying dynamic physiological processes in live neurons using fluorescence recovery after photobleaching. J Neurochem $126: 213-222$

3. Wade MH, Trosko JE, Schindler M (1986) A fluorescence photobleaching assay of gap junctionmediated communication between human cells. Science 232:525-528

4. Abbaci M, Barberi-Heyob M, Blondel W et al (2008) Advantages and limitations of commonly used methods to assay the molecular permeability of gap junctional intercellular communication. Biotechniques 45(33-52):56-62

5. Kuzma-Kuzniarska M, Yapp C, Pearson-Jones TW et al (2014) Functional assessment of gap junctions in monolayer and three-dimensional cultures of human tendon cells using fluorescence recovery after photobleaching. J Biomed Opt 19:15001, 1-15001-7

6. Abbaci M, Barberi-Heyob M, Stines JR et al (2007) Gap junctional intercellular communication capacity by gap-FRAP technique: a comparative study. Biotechnol J 2:50-61

7. Waggett AD, Benjamin M, Ralphs JR (2006) Connexin 32 and 43 gap junctions differentially modulate tenocyte response to cyclic mechanical load. Eur J Cell Biol 85:1145-1154 